\title{
A Rare Tumor: Small Cell Prostate Carcinoma Case Report
}

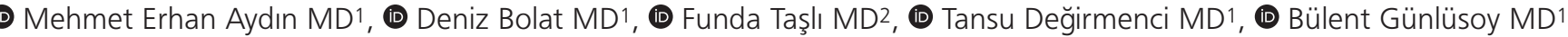 \\ 'University of Health Sciences, Izmir Bozyaka Training and Research Hospital, Clinic of Urology, Izmir, Turkey \\ ${ }^{2}$ University of Health Sciences, Izmir Bozyaka Training and Research Hospital, Clinic of Medical Pathology, Izmir, Turkey
}

\begin{abstract}
Prostate small cell carcinoma is a rare and aggressive tumor. They can be distinguished from classic prostate adenocarcinoma by features such as lack of prostate-specific antigen secretion, failure to respond to androgen suppression therapy, osteolytic bone lesions, and visceral metastasis. Herein, we present a case of previously diagnosed prostate adenocarcinoma that transformed to prostate small cell carcinoma, together with a discussion of the current literature.
\end{abstract}

Keywords: Prostate cancer, small cell carcinoma, prostate-specific antigen, survival

\section{Introduction}

Prostate cancer is the second most common cancer in men (1). Prostatic small cell carcinoma (PSCC) is a rare and aggressive tumor. It accounts for $0.5-2 \%$ of all prostate cancers (2) and the mean age at detection is 65 years (3).

Although Wenk et al. (4) first described PSCC in 1977, their biological behavior remains unclear (5). PSCC has features unlike prostate adenocarcinoma such as lack of prostate-specific antigen (PSA) secretion, nonresponse to androgen suppression therapy, and formation of osteolytic bone lesions and visceral metastases, and follows an aggressive course (6).

Due to the absence of androgen receptors in PSCC cells, it is hormone-resistant and is currently treated similarly to small cell lung cancer (5). Prognosis is poor, with only a few cases of complete remission reported in the literature (7).

In this article, we describe the development of PSCC in a patient who was diagnosed with prostate adenocarcinoma but could not be followed regularly, and discuss the case in light of the literature.

\section{Case Presentation}

An 87-year-old male patient was referred to our clinic in May 2017 due to bladder perforation that occurred during passive transurethral resection of the prostate (TURP) at another center. According to the patient's history, TURP performed in 2013 due to PSA level of $84 \mathrm{ng} / \mathrm{mL}$ resulted in a diagnosis of prostate adenocarcinoma (Gleason score: $4+3$ ) and hormone therapy was recommended, but his treatment adherence was poor.

The discharge report from the other medical center indicated that prior to TURP, the patient's prostate was grade- 3 in size, hard and fixed on rectal examination, PSA was $>100 \mathrm{ng} / \mathrm{mL}$, and urinary system ultrasound (US) showed grade- 2 dilation in the collecting systems of both kidneys and a $40 \times 21 \mathrm{~mm}$ mass in the bladder base that was evaluated as a prostatic invasion of the bladder.

Abdominal US performed during TURP due to the development of abdominal distension showed free fluid in the abdomen. A peroperative drain was placed in the abdomen and the patient was referred to our clinic for further examination and treatment. On physical examination, the abdomen was painless 
with no distention or rebound. Laboratory results showed creatinine level of $3.4 \mathrm{mg} / \mathrm{dL}$, leukocyte count of $10,310 / \mathrm{mm}^{3}$, hemoglobin level of $12.9 \mathrm{~g} / \mathrm{dL}$, and the other laboratory values were within normal range. Under local anesthesia, the patient underwent bilateral US-guided percutaneous nephrostomy. The patient's leukocyte and creatinine values returned to normal range during follow-up. The abdominal fluid resolved and the drain was removed, followed by the urethral catheter.

The pathology report for the TURP indicated $90 \%$ small cell carcinoma and $10 \%$ prostate adenocarcinoma (Gleason score: $4+3$ ). The tumor showed occasional irregular cribriform pattern in the acinar cell carcinoma areas, while small cell carcinoma morphology showing solid layering was seen in the large areas. Tumor cells in these areas had relatively uniform, narrow cytoplasm and coarse chromatin pattern. In immunohistochemical staining, the small cell carcinoma areas were CD56, chromogranin, synaptophysin, thyroid and transcription factor-1 (TTF-1) positive; cytokeratin focal positive; PSA, prostate-specific acid phosphatase (PSAP), and alpha-

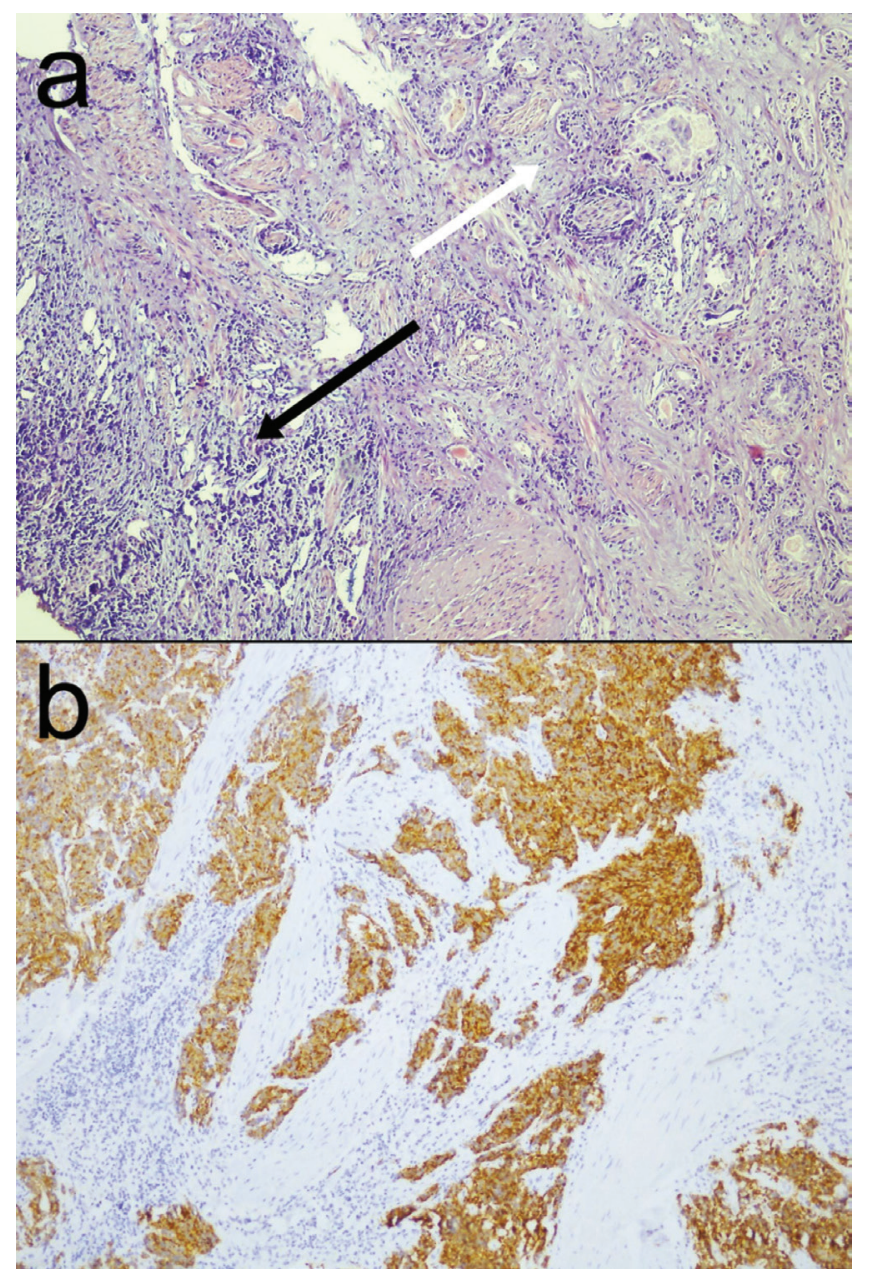

Figure 1. a) Prostate adenocarcinoma with perineural invasion in the upper right area, prostate small cell carcinoma in the lower left (hematoxylin and eosin; 100x); b) Cytoplasmic synaptophysin staining in tumor cells showing diffuse stratification (immunohistochemistry, synaptophysin; 100x) methylacyl-coenzyme A racemase (AMACR) negative, and Ki67 index was 80\% (Figures 1 and 2). No metastatic lesions were detected in contrast-enhanced thoracic and whole-body computed tomography scans conducted for staging purposes. Whole-body bone scintigraphy (WBBS) revealed multiple areas of increased uptake in the thoracic and lumbar vertebrae, the costa, and both acetabulums, and the patient was started on maximal androgen blockade (MAB) therapy and referred to the medical oncology department for chemotherapy. It was learned that the patient did not present to medical oncology and died 2 months later.

\section{Discussion}

Histologically, most prostate cancers are adenocarcinomas originating from prostate glandular cells. PSCC is a rare and aggressive malignancy of the prostate, with a mean age at diagnosis of 65 (3).

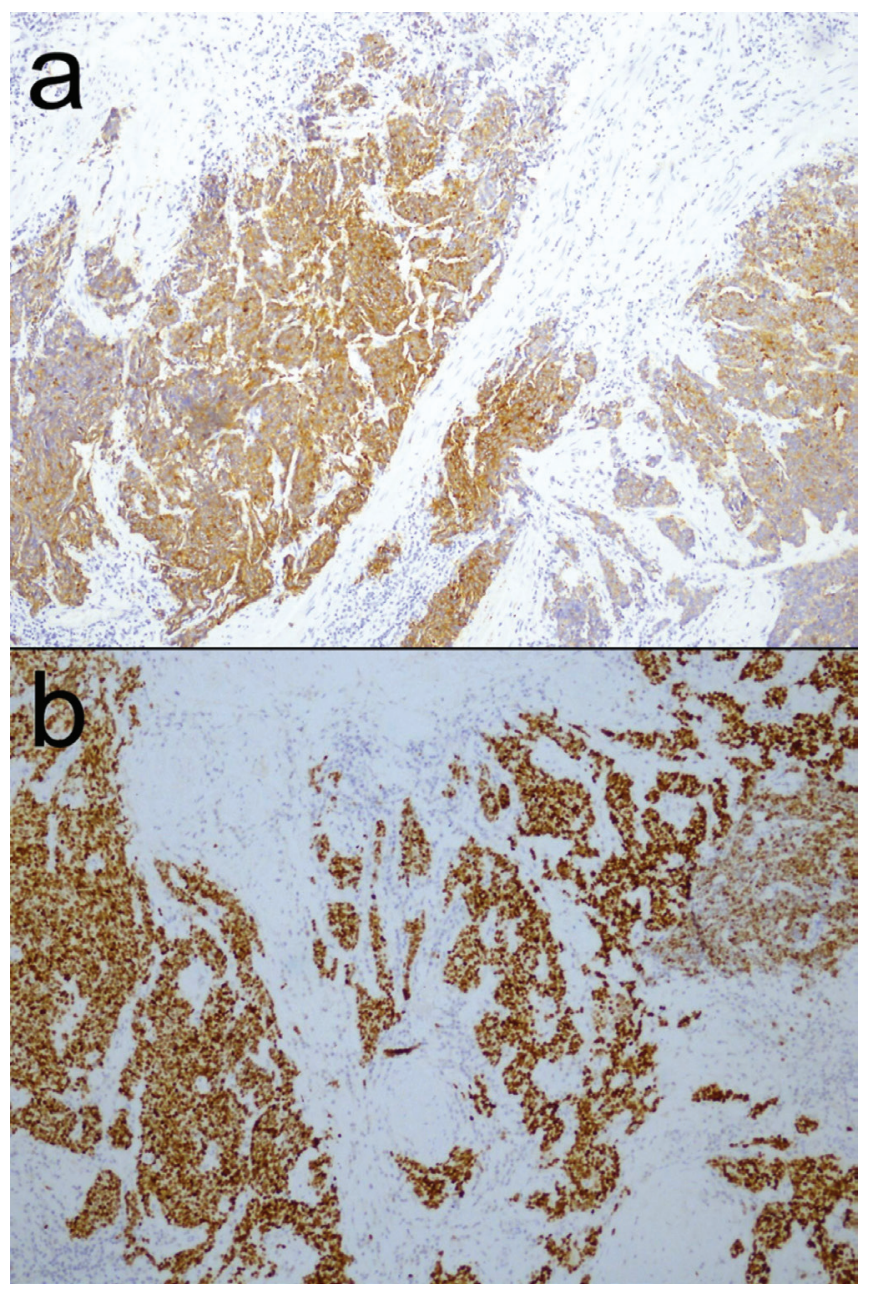

Figure 2. a) Cytoplasmic chromogranin staining in the prostatic small cell carcinoma component (immunohistochemistry, chromogranin; 100x); b) nuclear thyroid and transcription factor-1 staining in the prostatic small cell carcinoma component (immunohistochemistry, thyroid and transcription factor-1; 100x) 
There are various theories regarding the origins of PSCC. In patients previously diagnosed with prostate adenocarcinoma, it is believed to arise due to adenocarcinoma cell transformation or to neuroendocrine cell proliferation induced by antiandrogen therapy, and due to neuroendocrine differentiation after radiotherapy in some patients (8). Another theory suggests that the neuroendocrine component originates from malignant transformation of normal prostate cells or pluripotent epithelial cells (5).

Approximately $50 \%$ of patients have pure small cell histology at time of diagnosis, while $25-50 \%$ have mixed prostate adenocarcinoma and small cell carcinoma. In about $25-40 \%$ of patients, initial diagnosis is adenocarcinoma only, but relapse occurs after hormone therapy as a combination of small cell carcinoma and adenocarcinoma (9). In the present case, the patient was initially diagnosed with prostate adenocarcinoma, underwent hormone therapy, and relapsed with PSCC and adenocarcinoma.

PSSC can be differentiated from classic adenocarcinomas through clinical behavior such as the formation of osteolytic bone and visceral organs metastasis and the presence of normal PSA level (5). In addition, staining of biopsy specimens is negative for androgen receptors (10). The most commonly used immunohistochemical markers for the tumor are neuronspecific enolase (NSE), chromogranin, synaptophysin, CD56, and TTF-1 (11).

Wang and Epstein (9) performed immunohistochemical studies on 95 patients with PSCC and showed that 92\% stained positive for CD-56 and $85 \%$ were positive for synaptophysin. These features facilitate the differentiation of PSSC from poorly differentiated acinar adenocarcinoma. Moreover, PSA and NSE were not detected in 14 hormone-resistant prostate carcinomas, while high serum chromogranin A levels were detected in 10 cases. Early detection of high chromogranin A levels may be an indicator to switch to a more aggressive treatment (9).

In the biopsy specimen obtained from our patient, some areas other than the prostate adenocarcinoma areas stained positive for CD56, chromogranin, synaptophysin, and TTF-1 but were negative for PSA, PSAP, and AMACR, leading to a diagnosis of PSCC in addition to prostate adenocarcinoma (Figure 1, 2).

These patients do not exhibit the expected increase in PSA level based on their prostate enlargement and the presence of metastatic disease (12). We attributed the elevated PSA in our patient to the prostate adenocarcinoma component of the tumor.

There is still no specific treatment for PSCC. The lack of androgen receptors in the PSCC cells renders hormone therapy ineffective (6). Prostate-limited tumors can be removed by radical prostatectomy (7).

Approximately $75 \%$ of patients have metastatic disease at the time of diagnosis. Metastases are usually in lymph node, liver, bone, lung, and brain, but there have also been occasional cases reported with metastases in locations such as the omentum, adrenal gland, and facial bones (13).

PSCC has similar morphological features to small cell lung cancer. Although a standard chemotherapy regimen has not been established for the treatment of metastatic PSCC, platinbased chemotherapy is generally used. Even if there is an initial response, this chemotherapy regimen is not an effective standard treatment (14). Radiotherapy is used to control local disease or as a palliative treatment option for patients with disseminated disease. Prognosis is poor, with an average survival of 6-17 months after diagnosis (15). In a study including 30 patients, Stein et al. (16) reported a remission of 54 months after chemotherapy in only 1 patient. The patients' mean survival time was 13 months (16). In another study, Cohen et al. (17) reported a 2-year survival rate of $97 \%$ for patients with adenocarcinoma versus 35\% for patients with PSCC, and noted that PSCC metastasizes rapidly.

In the present case, no visceral metastasis was detected in CT, while WBBS revealed multiple bone metastases, and the patient was started on MAB therapy and referred to medical oncology. The patient did not present to medical oncology and died a short time (2 months) after diagnosis.

PSCC is a rare and aggressive cancer and its clinical behavior differs from that of prostate adenocarcinoma. Although the treatment approach is similar to that used for small cell lung cancer, a standard treatment regimen not yet been established. PSCC becomes metastatic quickly and has a poor prognosis with short survival times, unlike classic prostate adenocarcinoma.

\section{Ethics}

Informed Consent: It wasn't taken.

Peer-review: Externally peer-reviewed.

\section{Authorship Contributions}

Surgical and Medical Practices: D.B., Concept: M.E.A., D.B., Design: T.D., Data Collection or Processing: F.T., Analysis or Interpretation: T.D., B.G., Literature Search: M.E.A., Writing: M.E.A.

Conflict of Interest: No conflict of interest was declared by the authors.

Financial Disclosure: The authors declared that this study received no financial support.

\section{References}

1. Ferlay J, Soerjomataram I, Dikshit R, et al. Cancer incidence and mortality worldwide: sources, methods and major patterns in GLOBOCAN 2012. Int J Cancer 2015;136: 359-386.

2. Helpap B, Köllermann J, Oehler U. Neuroendocrine differentiation in prostatic carcinomas: histogenesis, biology, clinical relevance, and future therapeutical perspectives. Urol Int 1999;62:133-138.

3. Demirtaş A, Sahin N, Oztürk F, et al. Small cell prostate carcinoma: a case report and review of the literature. Case Rep Urol 2013;2013:387931.

4. Wenk RE, Bhagavan BS, Levy R, et al. Ectopic ACTH, prostatic oat cell carcinoma, and marked hypernatremia. Cancer 1977;40:773-778.

5. López Cubillana P, Martínez Barba E, Prieto A, et al. Oat-cell carcinoma of the prostate. Diagnosis, prognosis and therapeutic implications. Urol Int 2001;67:209-212.

6. Wang HT, Yao YH, Li BG, et al. Neuroendocrine Prostate Cancer (NEPC) progressing from conventional prostatic adenocarcinoma: factors associated with time to development of NEPC and survival from NEPC diagnosis-a systematic review and pooled analysis. J Clin Oncol 2014;32:3383-3390. 
7. Albisinni S, De Nunzio C, Tubaro A. Pure small cell carcinoma of the prostate: A rare tumor. Indian J Urol 2012;28:89-91.

8. Durmaz $M$, Kilınç F, Buldu I, et al. Small cell carcinoma of the prostate in differential diagnosis - A case report. Gaziantep Med J 2016;22:160-163.

9. Wang W, Epstein JI. Small cell carcinoma of the prostate. A morphologic and immunohistochemical study of 95 cases. Am J Surg Pathol 2008;32:65-71.

10. Abrahamsson PA. Neuroendocrine differentiation in prostatic carcinoma. Prostate 1999;39:135-148.

11. Capizzello A, Peponi E, Simou N, et al. Pure small cell carcinoma of the prostate: a case report and literature review. Case Rep Oncol 2011;4:88-95.

12. Sella A, Konichezky M, Flex D, et al. Low PSA metastatic androgenindependent prostate cancer. Eur Urol 2000;38:250-254.

13. Têtu B, Ro JY, Ayala AG, et al. Small cell carcinoma of prostate associated with myasthenic (Eaton-Lambert) syndrome. Urology 1989;33:148-152.
14. Papandreou CN, Daliani DD, Thall PF, et al. Results of a phase II study with doxorubicin, etoposide, and cisplatin in patients with fully characterized small-cell carcinoma of the prostate. J Clin Oncol 2002;20:3072-3080.

15. Rubenstein JH, Katin MJ, Mangano MM, et al. Small cell anaplastic carcinoma of the prostate: seven new cases, review of the literature, and discussion of a therapeutic strategy. Am J Clin Oncol 1997;20:376-380.

16. Stein ME, Bernstein Z, Abacioglu U, et al. Small cell (neuroendocrine) carcinoma of the prostate: etiology, diagnosis, prognosis, and therapeutic implications--a retrospective study of 30 patients from the rare cancer network. Am J Med Sci 2008;336:478-488.

17. Cohen RJ, Glezerson G, Haffejee Z, Afrika D. Prostatic carcinoma: histological and immunohistological factors affecting prognosis. $\mathrm{Br}$ J Urol 1990;66:405-410. 